\title{
Correction to: Chemotherapy: a double-edged sword in cancer treatment
}

\author{
Nafiseh Behranvand ${ }^{1,2} \cdot$ Farzad Nasri $^{1,2} \cdot$ Reza Zolfaghari Emameh $^{3}\left[\right.$ Pouria Khani $^{4} \cdot$ Asieh Hosseini $^{5}$. \\ Johan Garssen $^{6,7} \cdot$ Reza Falak ${ }^{1,2}$ (1)
}

Published online: 28 August 2021

(c) Springer-Verlag GmbH Germany, part of Springer Nature 2021

\section{Correction to: Cancer Immunology, Immunotherapy https://doi.org/10.1007/s00262-021-03013-3}

The original version of this article unfortunately contained a mistake. Third author's name was incorrect. First name of this author was missed and an extra " $\mathrm{m}$ " is also inserted in his last name.

The first name of this author is "Reza" and his last name is "Zolfaghari Emameh" and ORCID \# is: 0000-0002-3253-7844

The original article has been corrected.

The original article can be found online at https://doi.org/10.1007/ s00262-021-03013-3.

Reza Falak

falak.r@iums.ac.ir

1 Immunology Research Center, Iran University of Medical Sciences, Tehran, Iran

2 Department of Immunology, School of Medicine, Iran University of Medical Sciences, Tehran, Iran

3 Department of Energy and Environmental Biotechnology, National Institute of Genetic Engineering and Biotechnology (NIGEB), Tehran, Iran

4 Department of Medical Genetics, Faculty of Medicine, Tehran University of Medical Sciences (TUMS), Tehran, Iran

5 Razi Drug Research Center, Iran University of Medical Sciences, Tehran, Iran

6 Division of Pharmacology, Faculty of Science, Utrecht Institute for Pharmaceutical Sciences, Utrecht University, Universiteitsweg 99, 3584 CG Utrecht, The Netherlands

7 Department Immunology, Danone Nutricia Research, Uppsalalaan 12, 3584 CT Utrecht, The Netherlands
Publisher's Note Springer Nature remains neutral with regard to jurisdictional claims in published maps and institutional affiliations. 